\title{
Drying Kinetics of Oca (Oxalis Tuberosa)
}

\section{Cinética de Secado de la Oca (Oxalis Tuberosa)}

\author{
D. F. Guamán Lozada, G. E. Carrera Oña, and A. F. Martinez Chavez
}

Facultad de Ciencias, Escuela Superior Politécnica del Chimborazo, Riobamba, Ecuador

I International Seminar of Livestock and Agroindustrial Production ESPOCH 2020

Corresponding Author:

G. E. Carrera Oña

gcarrera@espoch.edu.ec

Published: 2 September 2021

Production and Hosting by

Knowledge E

(c) D. F. Guamán Lozada et al. This article is distributed under the terms of the Creative Commons Attribution License, which permits unrestricted use and redistribution provided that the original author and source are credited.

\section{A OPEN ACCESS}

\section{Abstract}

One of the most important steps for agro-industrial industrialization is examining the kinetic behavior of products, to determine the ideal parameters to maintain their characteristics throughout the production chain. This study experimentally determined the drying kinetics of oca, an indigenous product of the Andean region, because there is no established information on the drying techniques of this tuber. The Andean region is the cradle of a large number of food crops that were domesticated and exploited by indigenous people thousands of years ago, even long before the expansion of the Inca civilization. In Ecuador, oca is perhaps the most important element of the daily diet, and an acceptable level of purity and hygiene has been reached. Oca provides fiber, essential amino acids and a low level of fat. In this study, a temperature of $60^{\circ} \mathrm{C}$ was set at the beginning of each experimental run. The weight of the oca slices was measured every $10 \mathrm{~min}$. The experiments were carried out in triplicate. Data were analyzed using StatGraphics software. Two drying periods were observed: the initial period of constant drying speed, where the removal of moisture corresponded to moisture not linked to the oca, while in the second stage, the internal moisture of the tuber was removed.

Keywords: kinetic, drying, goose.

\section{Resumen}

Uno de los pasos más importantes para la industrialización agroindustrial es el comportamiento cinético de productos, esto en especial para conocer cuáles son los parámetros ideales para mantener las características sensoriales durante toda la cadena de producción. El presente documento determina experimentalmente la cinética de secado para un producto autóctono de la Región Andina, como lo es la oca, puesto que no existe información sobre las técnicas de secado de este tubérculo. En el Ecuador se ha alcanzado un aceptable nivel de pureza e higiene, la Oca es quizás el elemento más importante de la dieta diaria. La región andina es cuna de un gran número de cultivos alimenticios que fueron domesticados y aprovechados por pueblos autóctonos hace miles de años, inclusive mucho antes de la expansión de la civilización Inca. Aporta fibra, aminoácidos esenciales y un nivel bajo en grasa. La experimentación para conocer las curvas que modelan el comportamiento cinético del secado, se llevó a cabo por triplicado; luego de realizar la recolección de datos, estos fueron analizados utilizando el software estadístico StatGraphics. donde se obtuvo el respectivo análisis de varianza y las curvas. En el estudio se configuró una temperatura de $60^{\circ} \mathrm{C}$ al inicio de cada corrida experimental, Se midió el peso de las rodajas de Oca cada 10 min, los datos fueron registrados y se procesó las tablas resultantes. En el estudio se pudo observar dos periodos de secado: Periodo inicial de velocidad de secado constante, donde la eliminación de humedad corresponde a la humedad no ligada a la oca, mientras en la segunda etapa se eliminó la humedad interna del tubérculo. 


\section{Introducción}

En el Ecuador se ha alcanzado un aceptable nivel de pureza e higiene, la Oca es quizás el elemento más importante de la dieta diaria. La región andina es cuna de un gran número de cultivos alimenticios que fueron domesticados y aprovechados por pueblos autóctonos hace miles de años, inclusive mucho antes de la expansión de la civilización Inca.

Con el transcurso del tiempo la importancia de algunos de estos cultivos andinos para la seguridad familiar ha adquirido importancia global, así como la papa. La mayoría sin embargo como la oca, melloco, mashua, son poco conocidos Internacionalmente y en los mismos países andinos, ignoradas las propiedades nutritivas de estos productos. La Oca constituye una parte de la herencia de nuestros antepasados, ha sido cultivada básicamente en la zona andina del Ecuador por sus condiciones climáticas y culturales que tienen una fuente en tierras llenas de minerales que favorecen a este tipo de cultivos.

La producción, el consumo y utilización de la oca mantienen una tendencia decreciente, a pesar que posee gran aporte de fibra, aminoácidos esenciales y un nivel bajo en grasa. Dejando de lado este tubérculo que para muchas personas está en el olvido incluso dentro de la gastronomía. Por este motivo es importante proporcionar información gastronómica nutricional y procesamiento en la obtención de harina de oca, la misma que servirá de enfoque fundamental de apertura al consumo dentro de la población.

Uno de los puntos esenciales dentro del procesamiento de la oca es considerar la cinética del secado por lo que a consideración se expone como fundamento técnico disminuir el agua disponible en el alimento para prevenir el desarrollo de microorganismos patógenos ya que también los alimentos secos pueden almacenarse durante periodos largos de tiempo.

Según Keqing [1] tenemos que los modelos de cinética de secado se pueden clasificar en:

Modelos Empíricos: Consideran al contenido de humedad inicial uniforme, el comportamiento de las piezas es isótropo y la resistencia interna es predominante al transporte del agua.

Modelos Difusionales: Basados en los mecanismos difusionales. Emplea como fuerza impulsora el gradiente de humedades.

Además, los tipos de cinética de secado se establecen según la aplicación teniendo entre los cuales la cinética del secado en los alimentos que permite conservarlos, obtener un producto de calidad además reducir el volumen y el peso disminuyendo los costos de empaque, almacenamiento y transporte. Un modelo matemático que describa este proceso podría ayudar a salvar obstáculos como daños al producto. [2].

En harinas el proceso de secado trata de reducir al $10 \%$ por lo tanto la temperatura no debe exceder $90^{\circ} \mathrm{C}$ lo cual permite reducir el crecimiento microbiano. Los secadores más conocidos son disco rotatorio y los secadores por tubos. 
La predicción de velocidad de secado es necesaria para la optimización de procesos. La modelización intenta establecer un modelo real para simplificar y delimitar el proceso de secado, [1].

\subsection{Oca}

En el continente americano existe abundante variedad de recursos naturales autóctonos, algunos de antiguo origen que por siglos han sido la base de la alimentación de las poblaciones nativas [3].

Entre los cultivos andinos se incluyen numerosas especies de tubérculos andinos, como es la oca; que destacan por su valor nutritivo. La mashua específicamente es un tubérculo que contiene almidón, vitaminas $\mathrm{C}$ y $\mathrm{B}$, proteínas, carbohidratos, fibras. La importancia que tiene la oca radica principalmente en el potencial rendimiento, asociado al alto contenido de glucosinolatos [4].

Se puede encarar el problema de la desnutrición, buscando alimentos alternativos de consumo. Se puede presentar a la harina de oca como una alternativa en la industria de la panificación. Tiene características muy favorables en la sustitución parcial del trigo [3].

La cinética del secado de un material no es más que la dependencia de la humedad del material y de la intensidad de evaporación con el tiempo o variables relacionadas con este, como la propia humedad o las dimensiones del equipo. Este es el mínimo contenido de humedad en la cual un material puede teóricamente ser secado. En una humedad característica ocurre un cambio en las condiciones de secado en las cuales pasa del secado a velocidad constante a la velocidad decreciente de secado. Contenido máximo de humedad higroscópica.

Esta es el equilibrio en el contenido de humedad del sólido cuando la humedad del ambiente está saturada [5].

Estudios anteriores y actuales indican que el tubérculo de la oca es una fuente importante de actividad antioxidante siendo comparado con frutas como la tuna y arándano que tiene un alto contenido de antioxidantes como flavonoides y polifenoles determinando por esto ser excelentes en términos de nutrición [4].

\subsection{Variables de secado}

Los alimentos presentan una transferencia de calor por convección y un contacto directo de la sustancia con el aire caliente en el cual tiene lugar la evaporación, se establece las condiciones básicas del proceso como son: temperatura, humedad relativa del aire de secado, flujo de aire, tamaño y forma del producto [4]. 


\section{Materiales y Métodos}

\subsection{Objeto de estudio}

Esta experimentación fue llevada a cabo en la facultad de ciencias pecuarias en el laboratorio de microbiología. Se empleó tubérculos de Oca en su estado de madurez fisiológica. La procedencia de los tubérculos fue del cantón Chambo de la provincia de Chimborazo. Los que fueron lavados para posteriormente cortarlos en rodajas de $3 \mathrm{~mm}$ de espesor y estos fueron colocados en orden sobre las bandejas de malla metálica del horno ubicado en las instalaciones de la Facultad de Ciencias Pecuarias de la Escuela Superior Politécnica del Chimborazo. El diagrama de flujo del proceso que se realizó se lo puede observar en la Figura 1.

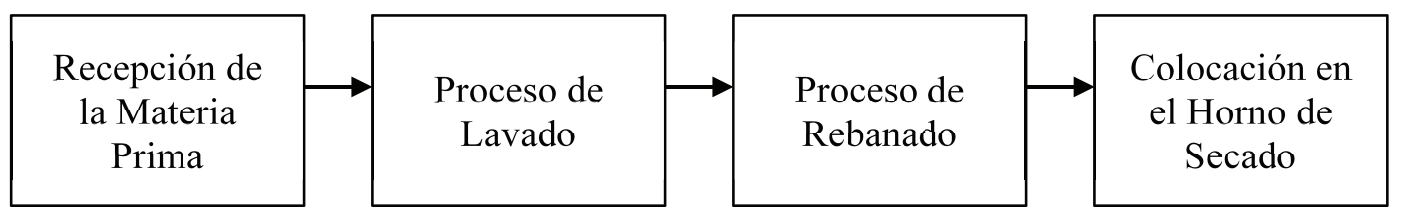

\section{Figure 1}

Flujograma del proceso hasta llevar al horno de secado las muestras de las ocas rebanadas en espesores de $3 \mathrm{~mm}$. Fuente: Propia.

\subsection{Análisis estadístico}

La experimentación se la llevó a cabo por triplicado, tratando de que la masa inicial de la materia húmeda esté dentro de un intervalo de $150 \mathrm{~g}$ a $160 \mathrm{~g}$. Luego de realizar la recolección de los datos, estos fueron analizados utilizando el programa estadístico StatGraphics Centurion. En donde a las muestras se las tomaron como un diseño experimental de un solo factor con muestras repetidas (relacionadas) para posteriormente obtener el respectivo Análisis de Varianza (ANOVA) y de esta manera probar la hipótesis de igualdad de residuos de dichas muestras.

Luego de comprobar dicha hipótesis, se procedió a realizar el promedio aritmético con cada una de las mediciones de las muestras, obteniendo así un solo conjunto de datos, los cuales nos sirvieron para obtener cada una de las gráficas de la cinética de secado.

\subsection{Curvas de secado}

En el estudio se configuró una temperatura de $60^{\circ} \mathrm{C}$. Y, al inicio de cada corrida experimental se verificó dicha temperatura. Enseguida se pesó la bandeja de soporte con las bandejas de malla metálica.

En el soporte de las bandejas se encontraba una balanza ubicada sobre la cámara de secado. Con la cual se midió la masa del conjunto esto es el soporte, las bandejas 
y las rodajas de la Oca cada 10 min, desde que comenzó el secado hasta que terminó (260 min).

Al finalizar, las rodajas fueron colocadas en una estufa a aproximadamente $80^{\circ} \mathrm{C}$ hasta obtener el peso constante final y de esta manera poder obtener el peso de la materia seca. Los datos experimentales se fueron registrando y procesando en tablas que se muestran en la sección de resultados. además, a partir de estos datos se pudo obtener las gráficas correspondientes a las curvas de la cinética de secado para luego poder realizar una descripción sobre las etapas presentes en la curva de secado de la Oca.

El contenido de masa (Ms) se lo obtuvo restando el peso total para cada tiempo de el peso de la bandeja. Además, para obtener el contenido de Humedad Residual $\left(X_{i}\right)$ en función del tiempo se utilizó la Ecuación 1.

$$
X_{i}=\frac{M s-M_{s f}}{M_{s f}}
$$

donde,

$$
M_{s f}=\text { Masa seca final. }
$$

La tasa de variación de la humedad residual, denotada por $\frac{d y}{d t}$, se, a obtuvo aplicando Ecuación 2.

$$
\frac{d y_{i}}{d t}=\frac{X_{i+1}-X_{i}}{\Delta_{t}}
$$

donde,

$$
\begin{gathered}
X_{i+1}: \text { Humedad relativa en el tiempo } i+1 ; \\
X_{i}: \text { Humedad relativa en el tiempo } i \\
\Delta_{t}: \text { Incremento del tiempo }(10 \mathrm{~min}) .
\end{gathered}
$$

Y por último, el cálculo de la humedad relativa media $\left(Y_{m}\right)$ se la obtuvo con la Ecuación 3

La tasa de variación de la humedad residual, denotada por $\frac{d y}{d t}$, se,a obtuvo aplicando Ecuación 2.

$$
Y_{m}=\frac{X_{i+1}+X_{i}}{2}
$$

donde,

$$
\begin{gathered}
X_{i+1} \text { : Humedad relativa en el tiempo i }+1 ; \\
X_{i} \text { : Humedad relativa en el tiempo i. }
\end{gathered}
$$

Con cada uno de estos valores se realizaron las curvas de secado correspondiente, en donde se pudo identificar las etapas de la cinética de secado. 


\section{Resultados}

Las gráficas de la cinética de secado de las 3 muestras se pueden apreciar en la Figura 2.

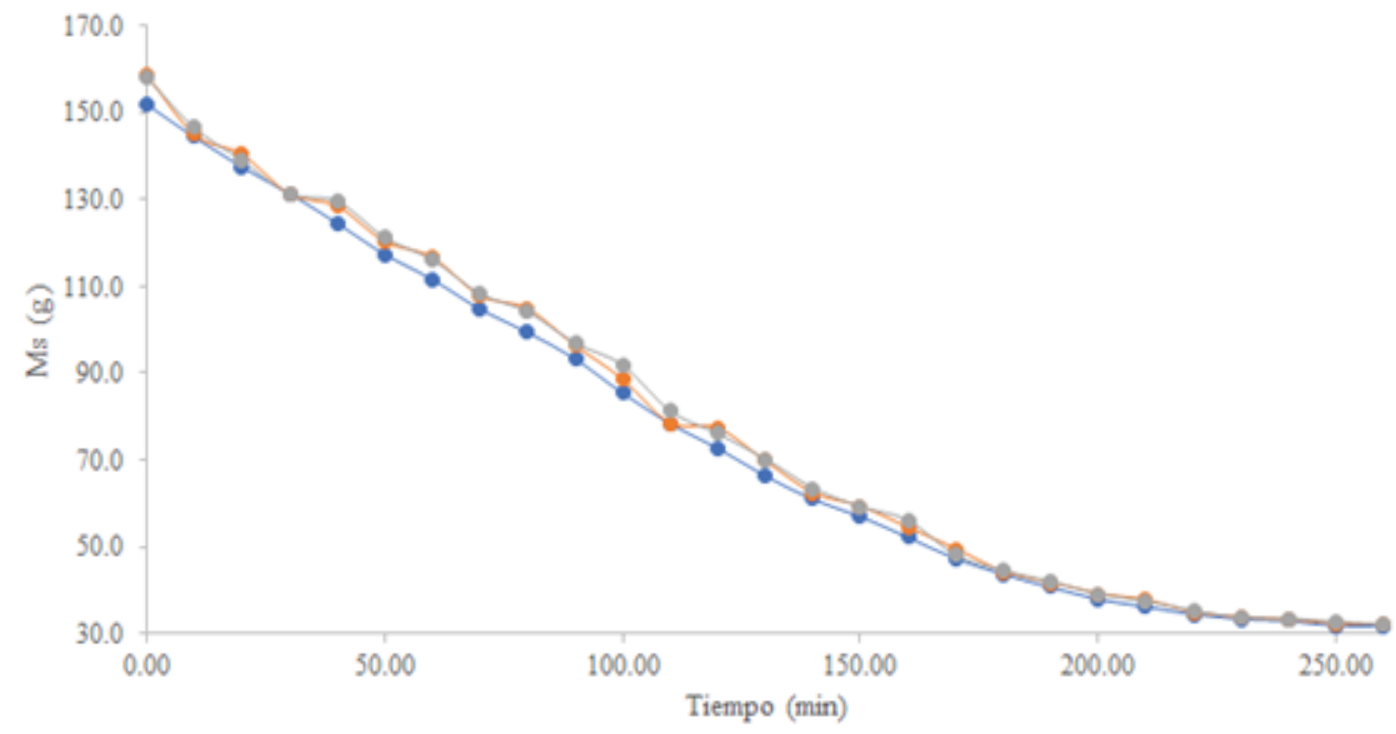

Figure 2

Materia seca de la oca en función del tiempo a $60^{\circ} \mathrm{C}$.

La tabla ANOVA correspondiente para verificar si las curvas de las 3 muestras son estadísticamente iguales se muestra a continuación en la Tabla 1.

\section{Table 1}

Tabla ANOVA.

\begin{tabular}{l|l|l|l|l|l|} 
Fuente & $\begin{array}{l}\text { Suma de } \\
\text { cuadrados }\end{array}$ & $\begin{array}{l}\text { Grados de } \\
\text { libertad }\end{array}$ & $\begin{array}{l}\text { Cuadrados } \\
\text { Medios }\end{array}$ & F & Valor - P \\
\hline Muestras & 3,40 & 2 & 1,704 & 0,38 & 0,6841 \\
\hline Tiempo & 1259999 & 26 & 4846,11 & 1087,73 & 0,0000 \\
\hline Error & 234,672 & 52 & 4,46 & & \\
\hline Total & 126234 & 80 & & & \\
\hline
\end{tabular}

De la Tabla 1, dado que el p-valor es un valor mayor a la significancia preestablecida de 0,05 , se concluye que las muestras son iguales.

Esto se lo puede apreciar a detalle con el gráfico de medias (Método LSD) que se presenta en la Figura 3.

Los datos promediados que obtenidos de la cinética de secado de la oca a una temperatura de $60^{\circ} \mathrm{C}$ son los que se muestran en la Tabla 2 .

De los datos mostrados en la Tabla 1 se logró obtener las curvas de la cinética de secado de la oca (Figuras 3 y 4). En la Figura 3 se puede apreciar las diferentes etapas del secado. Con un tiempo de aproximadamente $100 \mathrm{~min}$ a $60^{\circ} \mathrm{C}$ se puede observar que pasamos de la primera etapa de secado donde la pérdida de humedad es constante 


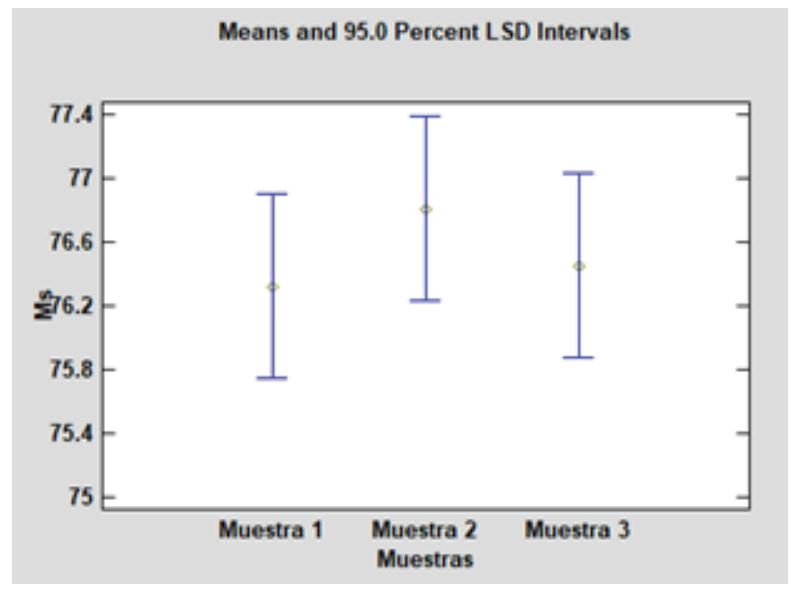

Figure 3

Gráfico de medias (método LSD).

a una etapa de $2 \mathrm{sec}$, donde la pérdida de la humedad es exponencial. Definiendo así la humedad crítica que es aproximadamente 1,65 g agua/g de oca seca.

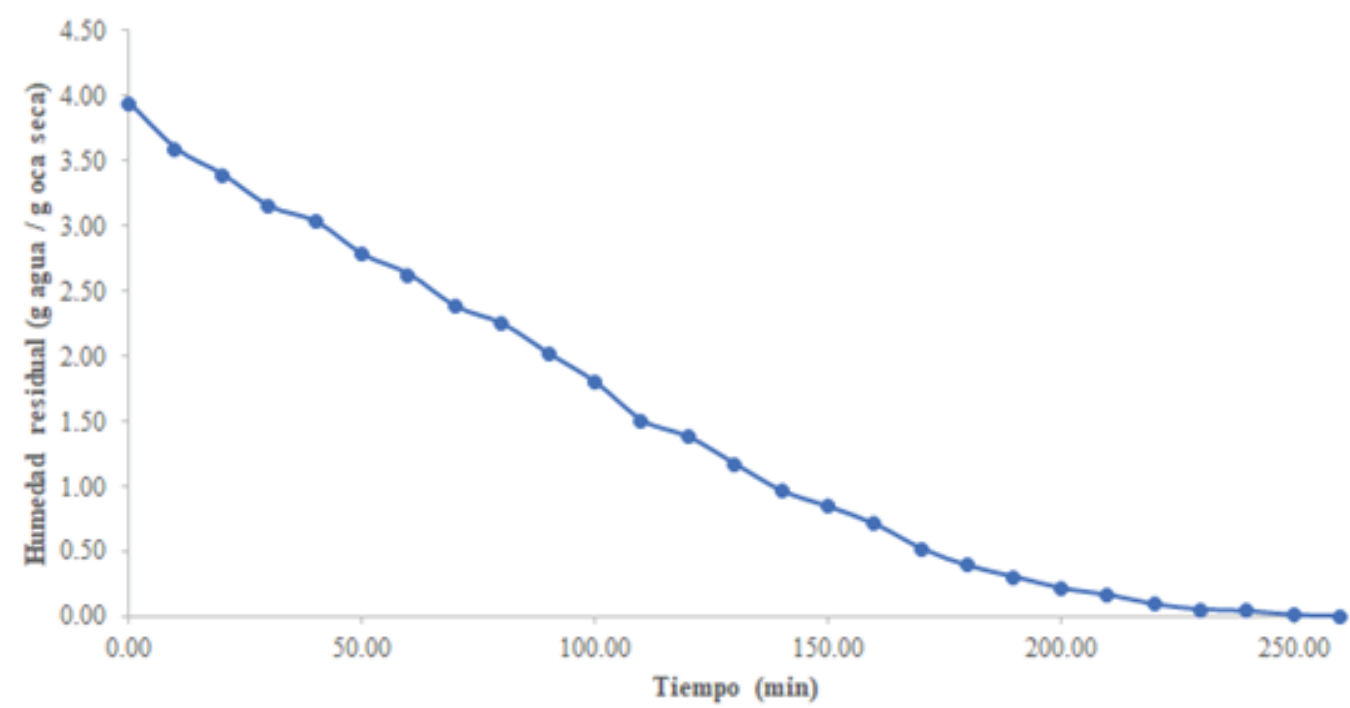

Figure 4

Humedad residual de la oca vs tiempo a $60^{\circ} \mathrm{C}$.

De la misma manera en la Figura 4 se presenta la variación de la humedad con relación a la humedad residual promedio, y al igual que en la Figura 1 se logra apreciar que la tasa de variación pasa a ser constante cuando la oca a alcanzado su humedad crítica a una humedad promedio de 1,65 (g agua/g oca seca), manteniendo una tasa de variación relativamente constante alrededor de 0,022. El tramo recto horizontal está relacionado con la eliminación del agua no ligada al producto. Mientras que en la etapa curva se observa claramente la eliminación del agua interna de la oca. 
Table 2

Datos experimentales de secado de rodajas de oca a $60^{\circ} \mathrm{C}$.

\begin{tabular}{|c|c|c|c|c|}
\hline Tiempo (min) & $\begin{array}{l}\text { Peso de las } \\
\text { rodajas al tiempo } \\
\text { (t) }\end{array}$ & $\begin{array}{l}\text { Humedad residual } \\
\text { (g agua / g oca seca) }\end{array}$ & $\begin{array}{l}\text { Tasa de variación de } \\
\text { la humedad (dY/dt) }\end{array}$ & $\begin{array}{l}\text { Humedad Promedio } \\
(\mathrm{Ym})\end{array}$ \\
\hline 0 & 156,50 & 3,94 & 0,035 & 3,76 \\
\hline 10 & 145,47 & 3,59 & 0,020 & 3,49 \\
\hline 20 & 139,17 & 3,39 & 0,024 & 3,27 \\
\hline 30 & 131,47 & 3,15 & 0,012 & 3,09 \\
\hline 40 & 127,73 & 3,03 & 0,025 & 2,90 \\
\hline 50 & 119,80 & 2,78 & 0,015 & 2,70 \\
\hline 60 & 114,93 & 2,63 & 0,025 & 2,50 \\
\hline 70 & 107,10 & 2,38 & 0,013 & 2,31 \\
\hline 80 & 102,90 & 2,25 & 0,023 & 2,13 \\
\hline 90 & 95,63 & 2,02 & 0,022 & 1,91 \\
\hline 100 & 88,70 & 1,80 & 0,029 & 1,65 \\
\hline 110 & 79,40 & 1,50 & 0,013 & 1,44 \\
\hline 120 & 75,40 & 1,38 & 0,021 & 1,27 \\
\hline 130 & 68,80 & 1,17 & 0,021 & 1,07 \\
\hline 140 & 62,30 & 0,97 & 0,012 & 0,90 \\
\hline 150 & 58,47 & 0,84 & 0,013 & 0,78 \\
\hline 160 & 54,20 & 0,71 & 0,019 & 0,62 \\
\hline 170 & 48,27 & 0,52 & 0,013 & 0,46 \\
\hline 180 & 44,10 & 0,39 & 0,009 & 0,35 \\
\hline 190 & 41,40 & 0,31 & 0,009 & 0,26 \\
\hline 200 & 38,63 & 0,22 & 0,005 & 0,19 \\
\hline 210 & 37,03 & 0,17 & 0,007 & 0,13 \\
\hline 220 & 34,77 & 0,10 & 0,004 & 0,08 \\
\hline 230 & 33,47 & 0,06 & 0,001 & 0,05 \\
\hline 240 & 33,10 & 0,04 & 0,003 & 0,03 \\
\hline 250 & 32,13 & 0,01 & 0,001 & 0,01 \\
\hline 260 & 31,87 & 0,01 & & \\
\hline
\end{tabular}

\section{Discusión}

En los procesos de secado por aire caliente, se pueden obtener diferentes periodos de secado: un periodo inicial de velocidad de secado constante (PVSC). Durante dicho periodo la superficie del producto está saturada de agua, y el secado ocurre a la temperatura de bulbo húmedo correspondiente al aire de secado.

La Cinética del secado se describe como un proceso de eliminación de substancias volátiles (humedad) para producir un producto sólido y seco. La humedad se presenta como una solución líquida dentro del sólido, es decir; en la microestructura del mismo. La transferencia de energía, en forma de calor, de los alrededores hacia el sólido 


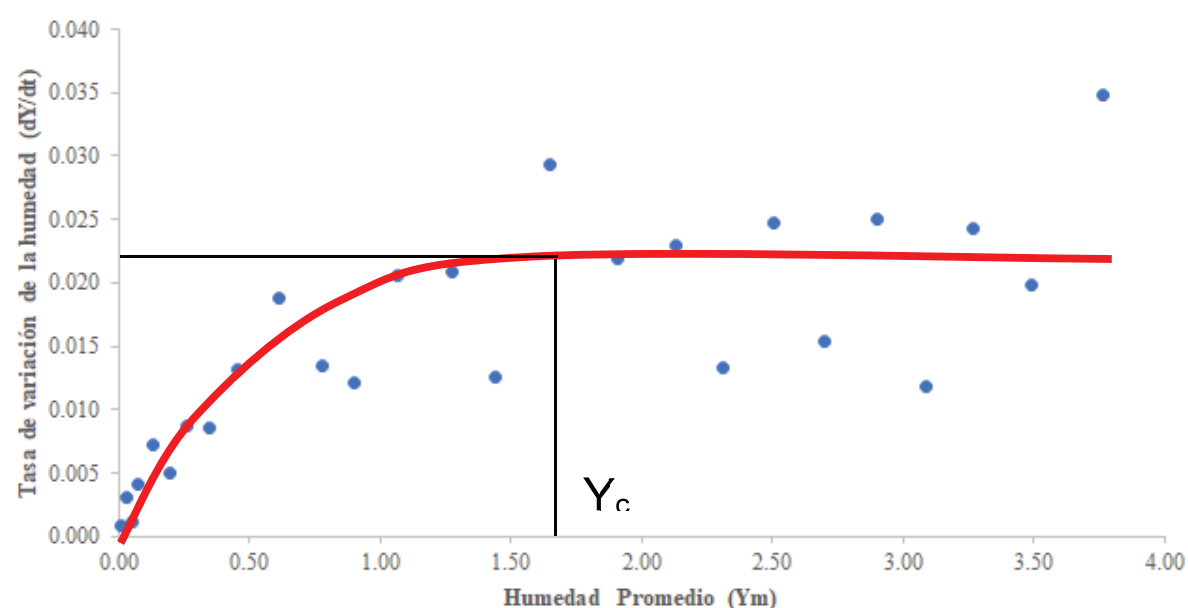

Figure 5

Tasa desecado de la oca vs Humedad promedio a $60^{\circ} \mathrm{C}$.

húmedo puede ocurrir como resultado de convección, conducción y/o radiación y en algunos casos se puede presentar una combinación de estos efectos [6].

La cinética de secado de un material no es más que la dependencia de la humedad del material y de la intensidad de evaporación con el tiempo o variables relacionadas con este, como la propia humedad o las dimensiones del equipo.

El secado de la materia prima de la oca tiene como objetivo eliminar agua no ligada en el producto a través de la transferencia de calor y de determinar la variación que tienen en la velocidad de secado y las variables del proceso tales como: temperatura, humedad de entrada, y la variación del tiempo para que este producto se pueda almacenar y conservar por un tiempo determinado antes del consumo.

El contenido de humedad de la oca varía enormemente. El agua es un constituyente primordial en la oca con un 83\%, según MINSA en el 2019 por lo que se optó por el método de secado en la estufa (deshidratado) con un peso final a las $12 \mathrm{hr}$ de 31,87 $\mathrm{g}$ habiendo determinado un porcentaje de humedad de la oca del 79,63\% ([156,5$31,87] / 156,5)$ lo que concuerda con investigaciones previas.

De las 3 muestras se puede apreciar en la Figura 2 que no existe mucha diferencia en los valores obtenidos en cada experimento, por lo que gráficamente se puede concluir que las muestras son iguales, y esto se comprueba mediante el análisis de varianza (Tabla 1) de muestras repetidas, además del gráfico de las diferencias de medias (Figure 4). Donde la masa inicial de la materia húmeda es de 156,6 g en promedio a temperatura de $60^{\circ} \mathrm{C}$

En la Figura 4 se puede apreciar las diferentes etapas del secado tomando en consideración la humedad residual. Con un tiempo de aproximadamente 250 min a $60^{\circ} \mathrm{C}$ se puede observar que pasamos de la primera etapa de secado donde la pérdida de humedad es exponencial a una etapa de secado 2 , donde la pérdida de la humedad es constante. la cual se observa a partir del minuto 100 aproximadamente. Es importante considerar un proceso de secado homogéneo en el cual la temperatura no 
debe ser muy elevada y no debería superar los $90^{\circ} \mathrm{C}$ para evitar alterar las propiedades organolépticas de sus componentes. En el proceso cinética de secado es importante la recirculación de aire en su interior para que el proceso de secado sea equilibrado en todos y cada uno de los componentes de la oca consiguiendo equilibrar y controlar el crecimiento bacteriano.

A partir de las gráficas y en función de los datos mostrados en la Tabla 2 indican que la humedad residual tendrá una humedad promedio de $1,65 \mathrm{~g}$ agua/g de oca seca obteniendo una variación de humedad constante de 0,022 dando como resultado que la materia prima habrá alcanzado su humedad crítica.

En los procesos de secado de aire caliente, se observan diferentes etapas. En nuestro estudio, al inicio observamos un descenso de humedad relativamente notorio hasta llegar a los 100,00 min donde se puede observar que hay mayor pérdida de humedad aproximadamente los 200,00 min donde se puede concluir que la velocidad con que se pierde a humedad a alcanzado valores cercanos a cero, por lo que se puede decir que las rodajas de oca, a partir de este momento han quedado libre de humedad. De esta manera podemos decir que obtenemos un producto sólido y seco libre de sustancias que puedan causar el crecimiento bacteriológico.

A partir de las curvas de cinética de secado ( $x$ vs $t$, $d x / d t$ vs $x$ ) como se observa en la Figura 2, que deben ser obtenidas a nivel de laboratorio, puede tenerse una idea del tiempo de secado, del consumo de energía, del mecanismo de migración de humedad, de las condiciones predominantes en la transferencia de calor y masa y de la influencia que tienen en la velocidad de secado las variables del proceso tales como: temperatura, humedad de entrada, velocidad del aire, etc. Por todo esto es que determinar las curvas de secado constituye uno de los objetivos fundamentales de este trabajo.

El modelo matemático logarítmico representa adecuadamente el proceso de secado de la oca. El aumento de la temperatura genera un aumento en la rapidez del proceso reduciendo el tiempo de secado con una tasa de pérdida de humedad aproximado del 22 a $26 \%$. La velocidad del aire tiene influencia sobre la rapidez en el secado, a mayor velocidad menor el tiempo para llegar al contenido de humedad de equilibrio deseado [7].

Otras investigaciones sugieren que que la deshidratación osmótica en las ocas (método no térmico) es mucho mejor que la deshidratación convencional, en cuanto a contenido de humedad y tiempos de secado [8]. Además de evitar la pérdida de características sensoriales como son el color, sabor y aroma.

La duración del de secado al aire libre depende de factores climáticos (temperatura, humedad relativa y velocidad del aire) y de las características propias de la oca, contenido de humedad, edad y sitio, por lo que no se puede estimar con exactitud un proceso normalizado [9].

Otros investigadores prefieren el uso de sistemas automatizados de secado, como por ejemplo el sistema de secado con aire conectivo a bajas temperaturas con la aplicación de ultrasonidos de potencia, el cual ha permitido ejecutar de manera sencilla 
y práctica la deshidratación de oca, puesto que, cuenta con sistema de control y monitoreo automatizado que permite registrar datos de frecuencia, temperatura, humedad y peso, en intervalos de tiempo programables [10].

\section{Conclusiones}

De los datos mostrados se logró obtener las curvas de la cinética de secado de la oca se puede apreciar las diferentes etapas del secado. Con un tiempo de aproximadamente $120 \mathrm{~min}$ a $60^{\circ} \mathrm{C}$ se puede observar que en la primera etapa de secado donde la pérdida de humedad es constante no obstante en una segunda etapa en la cual la pérdida de la humedad es exponencial. De esta manera definiendo así la humedad crítica que es aproximadamente $1,65 \mathrm{~g}$ agua/g de oca seca.

De la misma manera se presenta la variación de la humedad con relación a la humedad residual promedio, y al igual al tiempo aproximado de secado se logra apreciar que la tasa de variación pasa a ser constante cuando la oca a alcanzado su humedad crítica a una humedad promedio de 1,27 (g agua/g oca seca), manteniendo una tasa de variación relativamente constante alrededor de 0.027 . El tramo recto horizontal está relacionado con la eliminación del agua no ligada al producto. Mientras que al inicio se observa claramente la eliminación del agua de la superficie de las rodajas de la oca. Es así que será suficiente secar la pulpa de la oca durante aproximadamente 100 min en el secador de bandeja, para que la humedad en base húmeda de la pulpa baje hasta el aproximadamente $80 \%$, lo que facilitará $s \mu$ molienda y empaque seguro como harina de lúcuma.

Como conclusión final se deja la pauta para el estudio e investigación 'Cinética de secado de la Oca' para el desarrollo de técnicas eficaces de procesamiento y manejo.

Es necesario la implementación de sistemas automáticos de secado que permitan la recolección directa de cada una de los diferentes parámetros que intervienen y el control de los mismos.

\section{References}

[1] Keqing $X$. Optimización del secado por aire caliente de pera blanquilla. Universidad Politécnica de Valencia; 2004. Obtenido de: https://riunet.upv.es/bitstream/handle/10251/1834/tesisUPV2195.pdf? sequence=1

[2] Vega A, Andrés A, Fito P. Modelado de la cinética de secado del pimiento rojo (capsicum annuum I. CV lamuyo). Información Tecnológica. 2005.

[3] Espinosa P, Vaca R, Abad J, Crissman C. Raices y tuberculos andinos- Cultivos marginados en el Ecuador. Situacion actual y limitaciones para la produccion. Quito: Ediciones Abya Yala; 1996.

[4] Brack A, Suquilanda M. Diccionario Enciclopedico de las plantas utiles del Peru/Agricultura organica, alternativa tecnologica del futuro. Quito: UPS ediciones; 2004.

[5] Pantoja S, Alberto L. La Caracterización de la Mashua (tropaeolum tuberosum c.) en el Ecuador. Universidad Tecnológica Equinoccial; 2012.

[6] Baker DR. Variables de secado. Mosfata; 1997.

[7] Cova L. Efecto de la estrategia de conservación en la calidad nutritiva de la harina de lombriz roja (Eisenia ssp.) a mediano plazo. Revista de la Facultad de Agronomía. 2009.

[8] Carpio T, E F. Universidad Nacional del Altiplano Puno. 2009 August 13. Obtenido de: http://repositorio. unap.edu.pe/handle/UNAP/3351 
[9] Arango JA. Google academico. 2006 May 30. Obtenido de: http://revistas.utp.edu.co/index.php/ revistaciencia/article/view/6593

[10] Hancco, Mamani. Repositorio.unap. 2017. Obtenido de: http://repositorio.unap.edu.pe/bitstream/ handle/UNAP/3975/Hancco_Zaga_Jenny_Maribel_Mamani_Ordo\{\%\}C3\{\%\}B1ez_Pedro_Nicoyani.pdf? sequence $=1 \&$ isAllowed $=y$ 